\title{
Robot-assisted thoracoscopic surgery for mediastinal masses: a single-institution experience
}

\author{
Kai Chen, Xianfei Zhang, Runsen Jin, Jie Xiang, Dingpei Han, Yajie Zhang, Hecheng Li \\ Department of Thoracic Surgery, Ruijin Hospital, Shanghai Jiao Tong University School of Medicine, Shanghai 200025, China \\ Contributions: (I) Conception and design: H Li, K Chen, R Jin; (II) Administrative support: H Li; (III) Provision of study materials or patients: J \\ Xiang, D Han, Y Zhang; (IV) Collection and assembly of data: X Zhang; (V) Data analysis and interpretation: K Chen; (VI) Manuscript writing: All \\ authors; (VII) Final approval of manuscript: All authors. \\ Correspondence to: Hecheng Li, MD, PhD. Department of Thoracic Surgery, Ruijin Hospital, Shanghai Jiao Tong University School of Medicine, 197 \\ Ruijin 2nd Road, Shanghai 200025, China. Email: lihecheng2000@hotmail.com.
}

\begin{abstract}
Background: The objectives of this study were to evaluate the feasibility and safety of robot-assisted thoracic surgery (RATS) using the da Vinci robotic system for mediastinal mass resection and to describe the surgical approach for masses in different mediastinal regions.

Methods: We retrospectively reviewed data from 84 patients who underwent RATS for resection of a mediastinal mass from June 2015 to April 2019. The results were analyzed with descriptive statistics.

Results: The mediastinal mass was resected successfully in all patients, with one patient requiring conversion to conventional open surgery. There were no intraoperative complications. The most common diagnoses were thymoma $(n=17)$, bronchogenic cyst $(n=16)$, and schwannoma $(n=12)$. The surgical approach was determined by the location of the lesion and the planned extent of surgical resection. Resection was performed in the lateral decubitus position in 29 patients with posterior mediastinal tumor, semilateral decubitus position in 42 patients who only need resection of anterior mediastinal mass, and reverse Trendelenburg position in 13 patients undergoing thymectomy. The mean ( \pm standard deviation) operation time was $91.86 \pm 56.42 \mathrm{~min}$, duration of chest tube use was $1.83 \pm 0.93$ days, and postoperative hospital stay was $3.62 \pm 7.52$ days. Three patients $(3.5 \%)$ developed postoperative complications: one chylous fistula, which required reoperation, and one case each of atrial fibrillation and pulmonary infection, which were treated conservatively.

Conclusions: Our experience demonstrated that different surgical approaches of RATS are safe and feasible for mediastinal mass resection. An appropriate approach can be selected based on the mediastinal region in which the tumor is located.
\end{abstract}

Keywords: Mediastinum; robot-assisted thoracoscopic surgery; mediastinal mass

Submitted Jul 14, 2019. Accepted for publication Aug 22, 2019.

doi: $10.21037 /$ jtd.2019.08.105

View this article at: http://dx.doi.org/10.21037/jtd.2019.08.105

\section{Introduction}

Mediastinal masses account for approximately $3 \%$ of thoracic lesions (1). They are a heterogeneous group of lesions, which includes thymomas, bronchogenic cysts, neurogenic tumors, and thyroid tumors. Mediastinal tumors are derived from different germ layers, located in various positions of the thoracic cavity $(2,3)$. For decades, median sternotomy, posterolateral thoracotomy, and hemi-clamshell sternotomy have been the preferred surgical approaches for resection of mediastinal masses (4); however, substantial surgical trauma and morbidity are associated with these open procedures.

With the recent widespread use of minimally invasive techniques, such as video-assisted thoracic surgery (VATS) and robot-assisted thoracic surgery (RATS), there has 
been a progressive increase in the use of these techniques for excising mediastinal masses. VATS has been widely used for mediastinum tumor resection. VATS has been associated with shorter operation times, less intraoperative blood loss, less chest tube drainage, and shorter hospital stays than open procedures $(5,6)$. These benefits have been reported for both conventional and single-port VATS (7-10). Despite the advantages of VATS, the technique is limited by suboptimal visualization and inflexible instruments.

In the past decade, robotic surgery has become increasingly used in the field of surgery. In 2001, Yoshino et al. reported the first successful application of the da Vinci robotic system for thymectomy (11). The threedimensional, high-definition view with up to 10 times image magnification, 7 degrees of freedom of surgical instruments, and filtration of physiologic hand tremors (12) with robotic systems are ideal for removing mediastinal tumors in a small space. A few studies have described the advantages of robotic technology for mediastinal tumors. Robotic systems provide easy access to the small mediastinal space and allow safe removal of mediastinal tumors, with less trauma, fewer complications, shorter postoperative stays, and faster recovery than open surgery $(13,14)$.

Because of the diversity of mediastinal tumor locations, every treatment center has different perspectives regarding RATS for mediastinal masses, and no consensus has been reached. Many surgical methods and approaches have been described, including lateral decubitus, semi-lateral decubitus, and reverse Trendelenburg approaches (15-17).

Mediastinal mass resection with RATS has been performed in our institution since June 2015. In this report, we discuss our surgical plan and approach, which depend on the pathology and location of the mediastinal mass. We also present the short-term clinical outcomes of patients undergoing surgical resection of mediastinal tumors with the da Vinci robotic system at our institution.

\section{Methods}

We retrospectively analyzed data from 84 patients who underwent mediastinal mass resection with the robotic system in our hospital from June 2015 to April 2019. The main objective was to evaluate the feasibility and safety of RATS for mediastinal mass resection. The secondary objective was to describe the surgical approach for resection of mediastinal masses in different sites using the da Vinci system.

\section{Preoperative evaluation}

Before surgery, the location and size of the mass, as well as the extent of invasion into surrounding organs, were assessed by enhanced chest computed tomography (CT) to determine the surgical method and approach. Cardiopulmonary, liver, kidney, and coagulation function were also evaluated. Patients with thymoma were assessed for symptoms of myasthenia gravis; when present, medical therapy was optimized before surgery.

\section{Surgical technique}

All surgical procedures were performed by the same surgical team using the da Vinci S system (Surgical Intuitive, Mountain View, CA, USA) with three arms. The patients received combined intravenous and inhalational general anesthesia and were intubated with a single-lumen endotracheal tube. During all procedures, carbon dioxide gas was insufflated into the thoracic cavity through the camera trocar at a flow rate of $8 \mathrm{~L} / \mathrm{min}$ and pressure of $8 \mathrm{mmHg}$.

The choice of surgical approach was determined by the location of the lesion and the planned extent of surgical resection. Patients with a mass in the posterior or superior mediastinum were positioned to elevate the operative side by 90 degrees (lateral decubitus position). If tumors in the anterior mediastinum had an intact capsule on CT scan, the intercostal approach with elevating the affected side by 45 degrees (semi-lateral decubitus position) was selected. If an anterior mediastinal mass exhibited no obvious capsule on $\mathrm{CT}$, or if a thymoma required bilateral thymic tissue dissection, the subxiphoid approach (reverse Trendelenburg position) was preferred.

At the start of the operation, the position of the assistant's hole was marked, but usually the hole was not created unless the surgeon found it difficult to complete the surgery alone. After completing the resection, all specimens were removed with an Endoscopic bag (Maslech Medical Technology Co. Ltd., Jiangsu, China) through the camera trocar incision and sent for pathological examination. A single $20 \mathrm{Fr}$ chest drainage tube was inserted in the thoracic cavity on the side of the surgical approach.

\section{Intercostal approach}

\section{Anterior mediastinal lesions}

Patients were placed in the semi-lateral position with 45 degrees elevation of the side to be approached, with a 


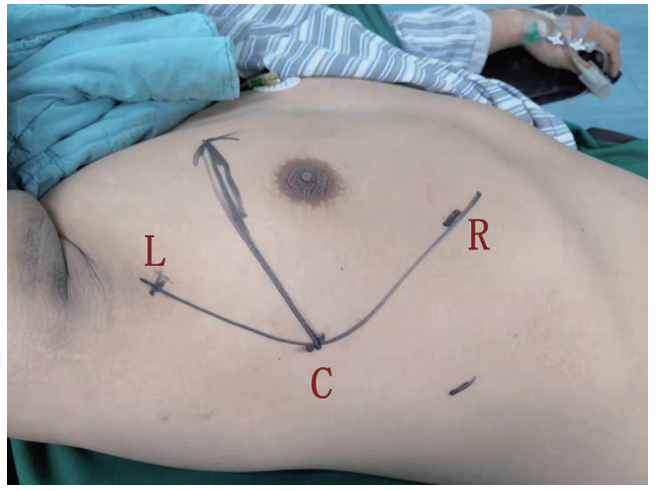

Figure 1 Placement of trocars for surgery in the semi-lateral decubitus position. $\mathrm{C}$, camera port; $\mathrm{L}$, left arm port; $\mathrm{R}$, right arm port.

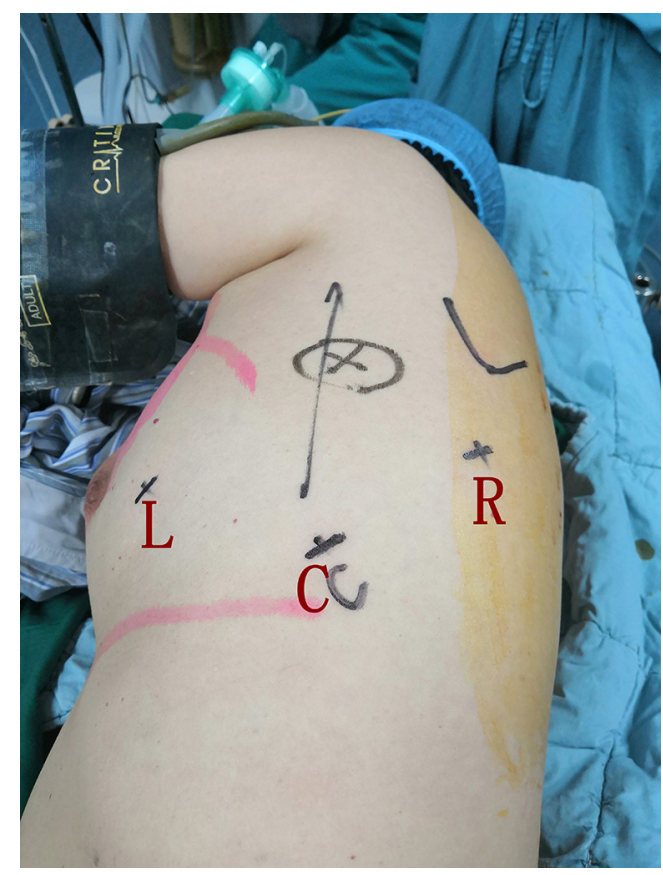

Figure 2 Placement of trocars for surgery in the lateral decubitus position. C, camera port; L, left arm port; $\mathrm{R}$, right arm port.

pad positioned beneath the chest. The ipsilateral arm was adducted and extended at the shoulder joint, moving the arm away from the surgical field. The robot (surgical cart) was docked from the ventral side (Figure 1). The camera trocar was first introduced in the fifth intercostal space on the midaxillary line. A zero-degree high-definition camera was used to visualize whether chest adhesions were present. One secondary trocar was inserted in the third intercostal space, and another trocar was placed in the fifth intercostal space on the anterior axillary line. A $12 \mathrm{~mm}$ trocar was placed as an auxiliary hole in the seventh intercostal space on the midaxillary line, when necessary. Careful dissection was accomplished using bipolar forceps (Surgical Intuitive, Mountain View, CA, USA) with the left arm and a cautery hook (Surgical Intuitive, Mountain View, CA, USA) with the right arm.

\section{Superior and posterior mediastinal lesions}

The patient was placed in a standard lateral decubitus position with 90 degrees elevation of the side to be approached. The robot was docked from the head side (Figure 2). The camera trocar was introduced at the ninth intercostal space on the midaxillary line. Two additional trocars were inserted, one in the sixth intercostal space on the anterior axillary region and another in the ninth intercostal space on the posterior axillary line. A $12 \mathrm{~mm}$ trocar was placed as an auxiliary hole in the eighth intercostal space on the anterior axillary line, when necessary. If the mass was located in the superior mediastinum, all trocars were positioned three intercostal spaces closer to the head. Grasping the specimen was usually performed using bipolar forceps with the left arm, and dissection and coagulation were performed using a cautery hook with the right arm.

\section{Subxiphoid approach}

Patients were placed in the reverse Trendelenburg position with 30 degrees of head elevation. The robot was docked from the head side (Figure 3). First, a $3 \mathrm{~cm}$ transverse incision was made below the xiphoid process, and the rectus abdominis was dissected from its attachment to the xiphoid process. The posterior aspect of the sternum was blindly detached using the finger. The camera port was inserted subxiphoid. Two additional trocars were inserted bilaterally below the costal arch. A cautery hook was used with the right arm, and bipolar forceps were used with the left arm. A chest tube was placed in the mediastinum through the lower hole of the xiphoid. If the mediastinal pleural was broken, the chest tube would be placed into the chest cavity through the hole under the rib arch.

\section{Follow-up}

Safety assessments included the identification of treatmentrelated complications within 30 days of surgery. The complications were categorized according to the ClavienDindo classification system (18). 


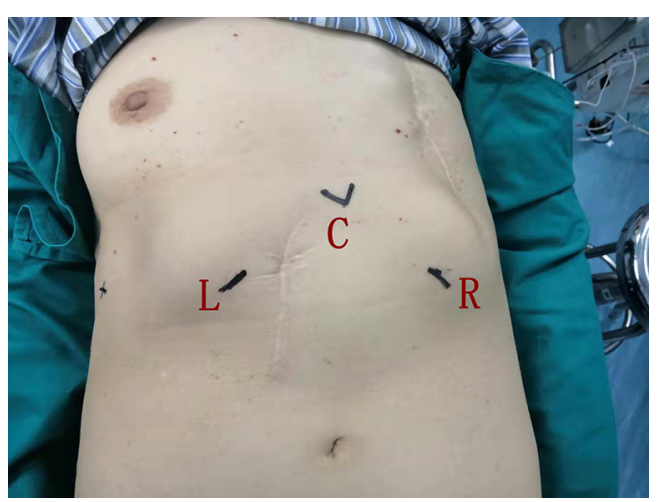

Figure 3 Placement of trocars for surgery in the reverse Trendelenburg position. C, camera port; L, left arm port; R, right arm port.

\section{Statistical analysis}

Baseline and outcome data (patient age, sex, and comorbidities; operation time; conversion rate; intraoperative blood loss; chest tube drainage volume and duration of use; complications; hospital length of stay; and mortality) were collected and analyzed using descriptive statistics. The results were expressed as frequency, mean, and standard deviation. Statistical analysis was performed using SSPSS 24.0 (IBM, Armonk, NY, USA).

\section{Results}

\section{Patient characteristics}

Of the 84 patients who underwent RATS, 44 were male and 40 were female (Table 1). Their mean age was $48.0 \pm 13.2$ years, and their comorbidities were as follows: hypertension, 15 patients (18\%); diabetes, 5 patients $(6 \%)$, depression, 1 patient (1\%); hyperlipidemia, 1 patient $(1 \%)$; hyperthyroidism, 1 patient (1\%); atrial fibrillation, 1 patient (1\%); and previous malignant tumor, 5 patients $(6 \%)$. The previous malignancies included breast cancer (2 patients; $2 \%)$, thymoma (1 patient; $1 \%)$, cervical cancer (1 patient; $1 \%)$, and melanoma (1 patient; $1 \%)$. The patient with a previous thymoma underwent RATS for recurrent tumor.

\section{Perioperative outcomes}

As shown in Table 2, no intraoperative complications occurred. In one patient, RATS was converted to open surgery because of a thymoma invading the surrounding organs (Masaoka-Koga stage $3 a$ ); it was difficult to
Table 1 Patient characteristics

\begin{tabular}{|c|c|}
\hline Variables & Value \\
\hline Sex, male/female & $44 / 40$ \\
\hline Age, years & $48.0 \pm 13.2$ \\
\hline Height, cm & $166.67 \pm 8.41$ \\
\hline Weight, kg & $66.83 \pm 11.23$ \\
\hline BMI, $\mathrm{kg} / \mathrm{m}^{2}$ & $23.93 \pm 2.74$ \\
\hline Smoking/non-smoking & $13 / 71$ \\
\hline \multicolumn{2}{|l|}{ Pulmonary function } \\
\hline $\mathrm{FEV}_{1}, \mathrm{~L}$ & $3.02 \pm 0.78$ \\
\hline MVV, L & $93.97 \pm 27.64$ \\
\hline $\mathrm{DLCO}, \mathrm{mL} \cdot \mathrm{kPa}^{-1} \cdot \mathrm{s}^{-1}$ & $6.94 \pm 1.70$ \\
\hline \multicolumn{2}{|l|}{ Comorbidities } \\
\hline Hypertension & 15 [18] \\
\hline Diabetes & $5[6]$ \\
\hline Depression & $1[1]$ \\
\hline Hyperlipidemia & $1[1]$ \\
\hline Hyperthyroidism & $1[1]$ \\
\hline Atrial fibrillation & $1[1]$ \\
\hline \multicolumn{2}{|l|}{ Previous malignancy } \\
\hline Breast cancer & 2 [2] \\
\hline Thymoma & $1[1]$ \\
\hline Cervical carcinoma & $1[1]$ \\
\hline Melanoma & $1[1]$ \\
\hline
\end{tabular}

Data are number, number (percentage), or mean \pm standard deviation. BMI, body mass index; DLCO, diffusing capacity for carbon monoxide; $\mathrm{FEV}_{1}$, forced expiratory volume in $1 \mathrm{sec}$; MVV, maximum voluntary ventilation.

completely remove the thymoma and thymic tissue with RATS in this case. Considering all patients, the mean tumor diameter was $5.03 \pm 2.46 \mathrm{~cm}$, mean operating time was $91.86 \pm 56.42 \mathrm{~min}$, mean blood loss was $89.12 \pm 303.39 \mathrm{~mL}$, mean total chest tube drainage was $281.40 \pm 333.70 \mathrm{~mL}$, mean duration of postoperative chest tube use was $1.83 \pm 0.93$ days, and mean postoperative hospital stay was $3.62 \pm 7.52$ days.

No mortality occurred. Morbidity (grade IIIII complications according to the Clavien-Dindo classification) occurred in 3 patients (3\%). A patient with hemangiolymphangioma developed a lymphatic fistula 
postoperatively, which was treated successfully with reoperation. This patient required hospitalization for 73 days. Two additional patients, one with a pulmonary infection and one with atrial fibrillation, were treated successfully with medications.

\section{Surgical position and approach}

As mentioned in Methods, the surgical approach was determined by the location of the lesion and the planned extent of surgical resection in our institution. Of the 84 operations, 29 were performed in the lateral decubitus position, 42 in the semi-lateral decubitus position, and 13 in the reverse Trendelenburg position (Table 3).

Table 2 Perioperative outcomes

\begin{tabular}{lc}
\hline Variables & Value \\
\hline Tumor diameter, cm & $5.03 \pm 2.46$ \\
Total operation time, min & $91.86 \pm 56.42$ \\
Intraoperative blood loss, $\mathrm{mL}$ & $89.12 \pm 303.39$ \\
Volume of chest tube drainage, $\mathrm{mL}$ & $281.40 \pm 333.70$ \\
Duration of chest tube use, days & $1.83 \pm 0.93$ \\
Postoperative complications & \\
Chylous fistula & $1[1]$ \\
Pulmonary infection & $1[1]$ \\
Atrial fibrillation & $1[1]$ \\
Duration of postoperative hospital stay, & $3.62 \pm 7.52$ \\
days & \\
\hline
\end{tabular}

Data are number (percentage) or mean \pm standard deviation.
The mean operation time was shortest in the lateral position $(86.03 \pm 70.23 \mathrm{~min})$ and longest in the reverse Trendelenburg position $(107.45 \pm 27.18 \mathrm{~min})$. However, the differences among these groups were not statistically significant. Moreover, mean blood loss was highest in the lateral decubitus position $(143.59 \pm 513.36 \mathrm{~mL})$, while in the semi-lateral decubitus position it was similar to that in reverse Trendelenburg position. Mean total volume of chest tube drainage, duration of chest tube use, and length of postoperative hospital stay were similar in the three positions. Collectively, these perioperative outcomes were comparable among different surgical approaches.

\section{Patbology data}

On postoperative histological examination, the most common tumors were thymomas (17 patients; 20\%), bronchogenic cysts (16 patients; 19\%), and schwannomas (12 patients; 14\%) (Table 4). Table 5 shows the World Health Organization (WHO) classes and Masaoka-Koga stages of the thymomas, as well as the associated comorbidities in patients with these tumors.

\section{Discussion}

With its advantages of high-definition, three-dimensional views, articulating endo-wristed instruments, and tremorfiltering capabilities (19), robotic-assisted surgery has become popular. Since mediastinal mass resection via RATS was first reported $(11,20)$, the technique has been increasingly used worldwide for a number of different types of mediastinal masses (21-24).

Table 3 Outcomes according to operative position

\begin{tabular}{lccc}
\hline Variables & Lateral decubitus & Semi-lateral decubitus & Reverse trendelenburg \\
\hline Number & $29(34.52)$ & $42(50.00)$ & $13(15.48)$ \\
Tumor diameter, cm & $4.39 \pm 2.32$ & $5.22 \pm 2.25$ & $5.88 \pm 3.16$ \\
Total operation time, min & $86.03 \pm 70.23$ & $91.07 \pm 52.52$ & $107.45 \pm 27.18$ \\
Intraoperative blood loss, $\mathrm{mL}$ & $143.59 \pm 513.36$ & $61.86 \pm 53.27$ & $55.70 \pm 32.05$ \\
Volume of chest tube drainage, $\mathrm{mL}$ & $257.54 \pm 233.73$ & $307.81 \pm 417.39$ & $249.34 \pm 206.31$ \\
Duration of chest tube use, days & $1.74 \pm 0.98$ & $1.96 \pm 1.00$ & $1.60 \pm 0.49$ \\
Duration of postoperative hospital & $2.72 \pm 1.03$ & $4.50 \pm 10.58$ & $2.76 \pm 0.92$ \\
stay, days & & & \\
\hline
\end{tabular}

Data are number (percentage) or mean \pm standard deviation. 
Table 4 Surgical pathology results

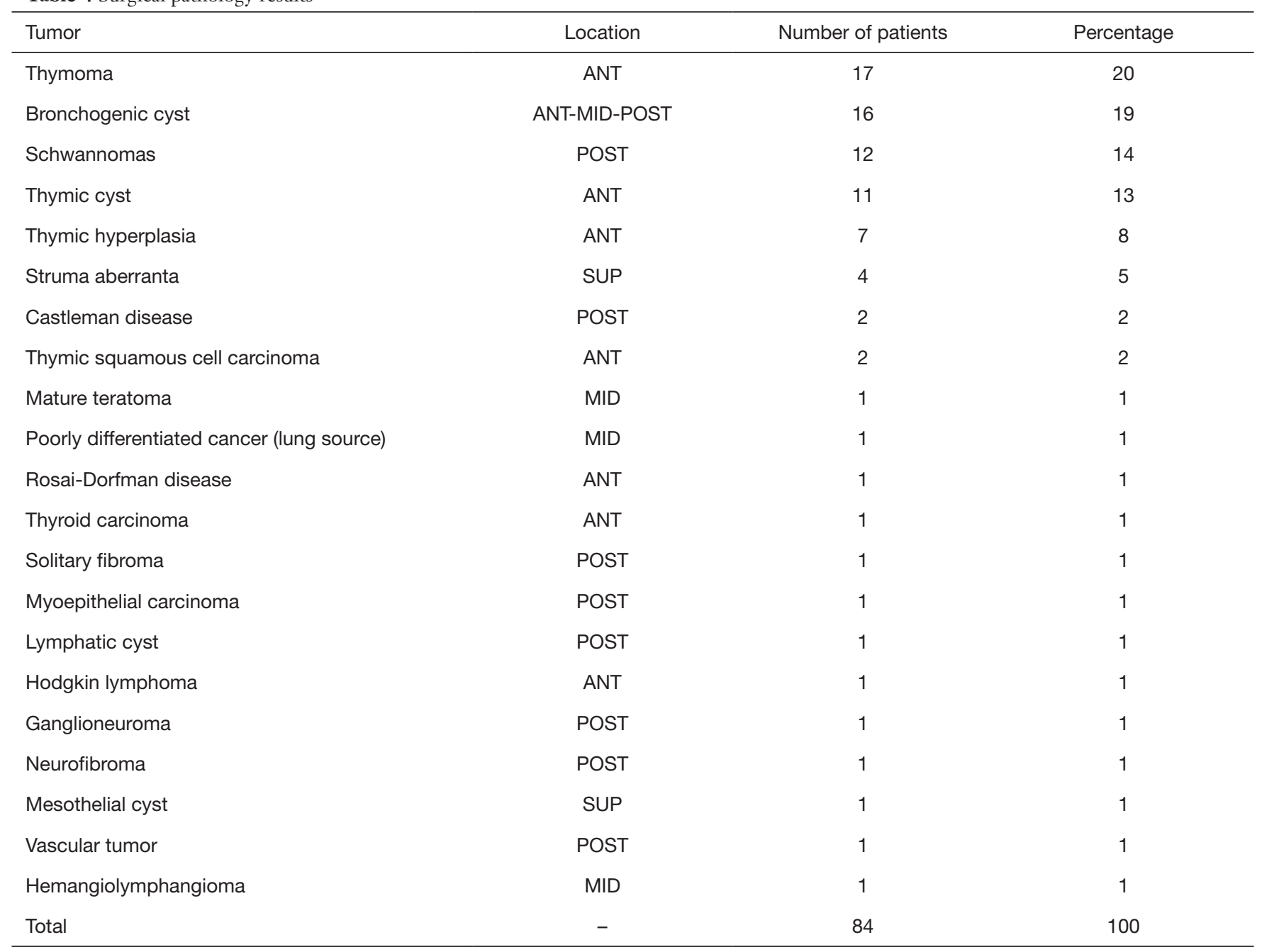

ANT, anterior mediastinum; MID, middle mediastinum; POST, posterior mediastinum; SUP, superior mediastinum.

Our experience with 84 patients shows that robotic resection of mediastinal tumors is safe and feasible. RATS was successfully completed in all except one patient, who had a Masaoka-Koga stage 3a (and WHO class B3) thymoma. Conversion to open surgery because of intraoperative complications was not required in any patient. We achieved relatively good results for all outcomes, including intraoperative blood loss, operation time, postoperative complications, chest tube drainage and duration of use, and length of hospital stay. Savitt et al. (25) and Toker et al. (21) previously demonstrated the safety and effectiveness of robotic-assisted surgery of the mediastinum. Seong et al. reported that RATS resulted in excellent early clinical outcomes in patients with relatively small mediastinal masses, when compared with a matched group of patients undergoing open surgery (26).

Preoperative evaluation to determine the robotic approach is very important for successful surgery (27). In our experience, RATS is the most efficient and effective when the surgical approach is based on the anatomical location of the mediastinal tumor. The most common method of mediastinal anatomical division is the fourcompartment model, which divides the mediastinum into superior, anterior, middle, and posterior compartments (28). In our study, 21 different pathologic types of mediastinal masses were located in various mediastinal compartments, and we chose the appropriate approach based on location.

For tumors in the posterior or superior mediastinum, the patient is placed in the standard lateral decubitus position; this results in the mass being located in line with the camera 
Table 5 WHO class and Masaoka-Koga stage of 17 thymomas and associated comorbidities

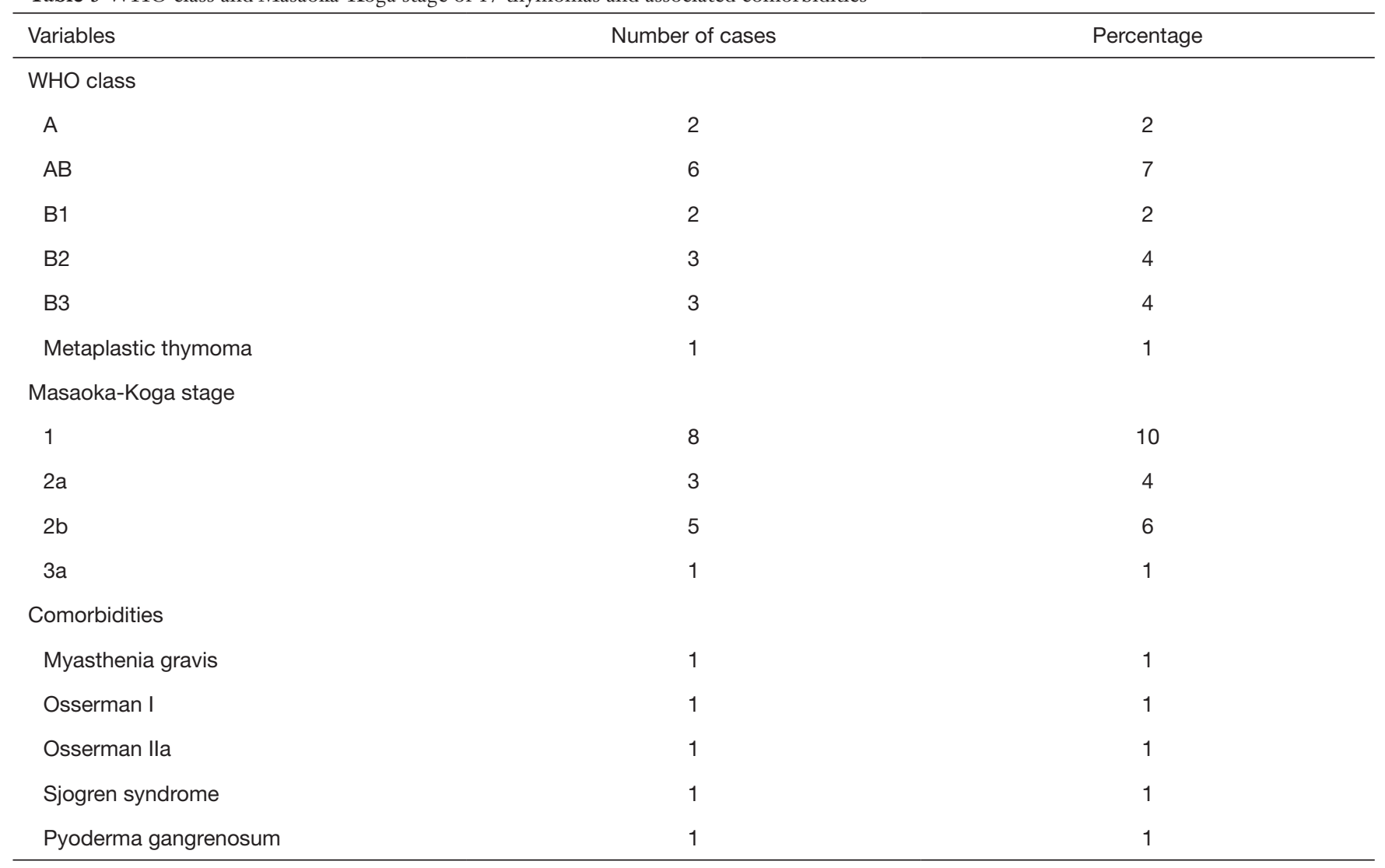

WHO, World Health Organization.

and surgical cart. We used this approach for 29 masses, including 12 neurogenic tumors, 2 Castleman disease masses, 3 thyroid tumors, and 12 other tumors. RATS is effective and safe for well-exposed neurogenic tumors (29), which contributed to our finding that the mean operation time was shortest in the lateral decubitus position. Broussard et al. also found that RATS was effective for posterior mediastinal pathology, when ideal trocar placement was used (17).

For tumors in the anterior or middle mediastinum, the patient is placed on the semi-lateral decubitus with 45 degrees elevation. Lateral decubitus positioning for these tumors would result in a poor field of vision and hindrance of right arm movements by the heart. Other surgeons have reported using a semi-lateral position with the affected side elevated at 30 degrees for anterior mediastinal masses and a lateral decubitus position for posterior mediastinal lesions (16,30). For patients with thymoma who require thymectomy, we choose the subxiphoid approach, while for those who have encapsulated intact thymoma or other anterior mediastinal masses, we prefer the intercostal approach. As some studies have demonstrated that patients with stage I-II without myasthenia gravis can be treated with thymomectomy (thymic tumor resection), or thymomectomy plus partial thymectomy (31-34).

Thymectomy is a unique procedure, which involves the removal of bilateral thymic tissue and fat. The subxiphoid approach is advantageous in thymectomy. The bilateral thymus tissue could be visible more clearly, that would help surgeons to achieve a complete resection of the tumor and thymus. However, we need to establish a space under the xiphoid to allow the camera and instruments to operate, whereas the intercostal approach can use the natural space of the chest cavity. Robotic thymectomy has been reported to be feasible and safe $(35,36)$. Our study included 10 patients with thymoma or thymic hyperplasia, two patients with a cyst mistaken for thymoma, and one patient with lymphoma in the thymic area. Although a needle biopsy was performed preoperatively, the lymphoma was misdiagnosed as thymoma because of the small tissue sample. Thymoma resection can be performed in either the semi-lateral decubitus or 
reverse Trendelenburg position. In our center, we initially performed robotic thymectomy in the semi-lateral decubitus position. However, after one patient required conversion to open thoracotomy because of unsatisfactory tumor exposure, we changed to using the subxiphoid approach in the reverse Trendelenburg position. The subxiphoid approach has been used by others for thymectomy to provide better visualization (37-39). With clearer exposure of the bilateral thymus tissue, it is much easier to achieve a complete resection of the tumor and thymus. Furthermore, if a thymic tumor cannot be removed by RATS in this position, the operation can be easily converted to a median sternotomy. Radkani et al. reported that RATS is feasible for mediastinal tumors in various mediastinal compartments (15).

Our study had some limitations. As a retrospective study, it was susceptible to selection bias. In addition, the number of patients was relatively small. Furthermore, we did not compare different approaches for tumors located in the same region, so we were unable to definitively demonstrate the superiority of surgical approach selection based on mediastinal anatomy. Different surgical approaches will be compared in future studies.

In conclusion, experience at our center demonstrated that different surgical approaches of RATS are safe and feasible for mediastinal masses. We suggest that the appropriate surgical approach-lateral decubitus, semi-lateral decubitus, or reverse Trendelenburg - can be based on the anatomical location of the mass within the mediastinum.

\section{Acknowledgments}

None.

\section{Footnote}

Conflicts of Interest: The authors have no conflicts of interest to declare.

Ethical Statement: The authors are accountable for all aspects of the work in ensuring that questions related to the accuracy or integrity of any part of the work are appropriately investigated and resolved. The study was approved by the Ethics Committee of Ruijin Hospital, Shanghai Jiao Tong University School of Medicine (No. 2019-161) and written informed consent was obtained from all patients.

\section{References}

1. Aroor AR, Prakasha SR, Seshadri S, et al. A study of clinical characteristics of mediastinal mass. J Clin Diagn Res 2014;8:77-80.

2. Takeda S, Miyoshi S, Akashi A, et al. Clinical spectrum of primary mediastinal tumors: a comparison of adult and pediatric populations at a single Japanese institution. J Surg Oncol 2003;83:24-30.

3. Davis RD Jr, Oldham HN Jr, Sabiston DC Jr. Primary cysts and neoplasms of the mediastinum: recent changes in clinical presentation, methods of diagnosis, management, and results. Ann Thorac Surg 1987;44:229-37.

4. Davenport E, Malthaner RA. The role of surgery in the management of thymoma: a systematic review. Ann Thorac Surg 2008;86:673-84.

5. Hwang SK, Park SI, Kim YH, et al. Clinical results of surgical resection of mediastinal teratoma: efficacy of video-assisted thoracic surgery. Surg Endosc 2016;30:4065-8.

6. Guo C, Mei J, Liu C, et al. Video-assisted thoracic surgery compared with posterolateral thoracotomy for mediastinal bronchogenic cysts in adult patients. J Thorac Dis 2016;8:2504-11.

7. Wu CY, Heish MJ, Wu CF. Single port VATS mediastinal tumor resection: Taiwan experience. Ann Cardiothorac Surg 2016;5:107-11.

8. Wu CF, Gonzalez-Rivas D, Wen CT, et al. Comparative short-term clinical outcomes of mediastinum tumor excision performed by conventional VATS and singleport VATS: is it worthwhile? Medicine (Baltimore) 2015;94:e1975.

9. Li Q, Sihoe A, Wang H, et al. Short-term outcomes of single- versus multi-port video-assisted thoracic surgery in mediastinal diseases. Eur J Cardiothorac Surg 2018;53:216-20.

10. Jiang $\mathrm{N}, \mathrm{Lu} \mathrm{Y}$, Wang J. Is single-port video-assisted thoracic surgery for mediastinal cystectomy feasible? J Cardiothorac Surg 2019;14:18.

11. Yoshino I, Hashizume M, Shimada M, et al. Thoracoscopic thymomectomy with the da Vinci computer-enhanced surgical system. J Thorac Cardiovasc Surg 2001;122:783-5.

12. Ashrafian H, Clancy O, Grover V, et al. The evolution of robotic surgery: surgical and anaesthetic aspects. $\mathrm{Br} \mathrm{J}$ Anaesth 2017;119:172-84. 
13. Cerfolio RJ, Bryant AS, Minnich DJ. Operative techniques in robotic thoracic surgery for inferior or posterior mediastinal pathology. J Thorac Cardiovasc Surg 2012;143:1138-43.

14. Zirafa CC, Melfi F. Robot-assisted surgery for posterior mediastinal mass. J Thorac Dis 2017;9:4929-31.

15. Radkani P, Joshi D, Barot T, et al. Robotic videoassisted thoracoscopy: minimally invasive approach for management of mediastinal tumors. J Robot Surg 2018;12:75-9.

16. Li H, Li J, Huang J, et al. Robotic-assisted mediastinal surgery: the first Chinese series of 167 consecutive cases. J Thorac Dis 2018;10:2876-80.

17. Broussard BL, Wei B, Cerfolio RJ. Robotic surgery for posterior mediastinal pathology. Ann Cardiothorac Surg 2016;5:62-4.

18. Seely AJ, Ivanovic J, Threader J, et al. Systematic classification of morbidity and mortality after thoracic surgery. Ann Thorac Surg 2010;90:936-42; discussion 942.

19. Ismail M, Swierzy M, Rückert JC. State of the art of robotic thymectomy. World J Surg 2013;37:2740-6.

20. Yoshino I, Hashizume M, Shimada M, et al. Video-assisted thoracoscopic extirpation of a posterior mediastinal mass using the da Vinci computer enhanced surgical system. Ann Thorac Surg 2002;74:1235-7.

21. Toker A, Özyurtkan MO, Kaba E, et al. Da Vinci robotic system in the surgery for mediastinal bronchogenic cyst: a report on five patients. J Vis Surg 2015;1:23.

22. Willems E, Martens S, Beelen R. Robotically enhanced mediastinal teratoma resection: a case report and review of the literature. Acta Chir Belg 2016;116:309-12.

23. Ward AF, Lee T, Ogilvie JB, et al. Robot-assisted complete thymectomy for mediastinal ectopic parathyroid adenomas in primary hyperparathyroidism. J Robot Surg 2017;11:163-9.

24. Linnik YA, Hoegemann Savellano D, Phillips JD, et al. A 49-year-old woman with right apical thoracic mass. Chest 2017;152:e133-8.

25. Savitt MA, Gao G, Furnary AP, et al. Application of robotic-assisted techniques to the surgical evaluation and treatment of the anterior mediastinum. Ann Thorac Surg 2005;79:450-5; discussion 455.

26. Seong YW, Kang CH, Choi JW, et al. Early clinical outcomes of robot-assisted surgery for anterior mediastinal mass: its superiority over a conventional sternotomy approach evaluated by propensity score matching. Eur J
Cardiothorac Surg 2014;45:e68-73; discussion e73.

27. Augustin F, Schmid T, Bodner J. The robotic approach for mediastinal lesions. Int J Med Robot 2006;2:262-70.

28. Susan S. Gray's anatomy: the anatomical basis of clinical practice. 41st ed. New York: Elsevier, 2016:976-80.

29. Pacchiarotti G, Wang MY, Kolcun JPG, et al. Robotic paravertebral schwannoma resection at extreme locations of the thoracic cavity. Neurosurg Focus 2017;42:E17.

30. Kajiwara N, Kakihana M, Kawate N, et al. Appropriate set-up of the da Vinci surgical system in relation to the location of anterior and middle mediastinal tumors. Interact Cardiovasc Thorac Surg 2011;12:112-6.

31. Nakagawa K, Asamura H, Sakurai H, et al. Does the mode of surgical resection affect the prognosis/recurrence in patients with thymoma? J Surg Oncol 2014;109:179-83.

32. Tseng YC, Hsieh CC, Huang HY, et al. Is thymectomy necessary in nonmyasthenic patients with early thymoma? J Thorac Oncol 2013;8:952-8.

33. Nakagawa K, Yokoi K, Nakajima J, et al. Is Thymomectomy alone appropriate for stage I (T1N0M0) thymoma? Results of a propensity-score analysis. Ann Thorac Surg 2016;101:520-6.

34. Onuki T, Ishikawa S, Iguchi K, et al. Limited thymectomy for stage I or II thymomas. Lung Cancer 2010;68:460-5.

35. Marulli G, Maessen J, Melfi F, et al. Multi-institutional European experience of robotic thymectomy for thymoma. Ann Cardiothorac Surg 2016;5:18-25.

36. Kamel MK, Rahouma M, Stiles BM, et al. Robotic thymectomy: learning curve and associated perioperative outcomes. J Laparoendosc Adv Surg Tech A 2017;27:685-90.

37. Suda T. Subxiphoid thymectomy: single-port, dual-port, and robot-assisted. J Vis Surg 2017;3:75.

38. Ruffini E, Filosso PL, Guerrera F, et al. Optimal surgical approach to thymic malignancies: New trends challenging old dogmas. Lung Cancer 2018;118:161-70.

39. Zheng Y, Cai YZ, Zhang HL, et al. Robotic transsubxiphoid extended thymectomy in a patient with thymoma-associated pemphigus. J Thorac Dis 2017;9:E565-9.

Cite this article as: Chen $\mathrm{K}$, Zhang X, Jin R, Xiang J, Han D, Zhang Y, Li H. Robot-assisted thoracoscopic surgery for mediastinal masses: a single-institution experience. J Thorac Dis 2020;12(2):105-113. doi: 10.21037/jtd.2019.08.105 